\title{
Gubernamentalidad neoliberal: miradas desde las intervenciones del Trabajo Social en el Gran La Plata, Argentina
}

Paula Mara Danel

Licenciada en Trabajo Social. Doctora en Trabajo Social

Universidad Nacional de La Plata y Conicet. La Plata, Argentina

https://orcid.org/0000-0001-7401-1720 • danelpaula@hotmail.com

Marcela Claudia Velurtas

Licenciada en Trabajo Social. Doctora en Trabajo Social

Universidad Nacional de La Plata. La Plata, Argentina

https:// orcid.org/0000-0002-7905-3724 $・$ mvelurtas@yahoo.com

\section{Agustina María Favero-Avico}

Licenciada en Trabajo Social. Magister en Derechos Humanos

Universidad Nacional de La Plata. La Plata, Argentina

https:/ / orcid.org/0000-0002-6307-3640 • agustinafavero@hotmail.com

\section{Resumen}

En el presente trabajo compartimos algunos avances de nuestra investigación vinculada a las intervenciones sociales del Estado entre los años 2016-2019, a partir de distintos campos de actuación del Trabajo Social en el Gran La Plata, Buenos Aires, Argentina. La categoría de intervención adquiere centralidad en esta investigación, en tanto resulta estructuradora de las indagaciones sostenidas sobre la relación entre ésta y las políticas sociales.

En los últimos años, hemos desarrollado distintas acciones investigativas orientadas a capturar la dinámica que adquiere en la actualidad la relación entre la intervención profesional de los trabajadores sociales y las políticas sociales en diferentes campos. Como parte de los resultados, advertimos que la intervención profesional ha adquirido, según las narrativas de las y los trabajadores sociales, algunas singularidades que nos dan pistas para caracterizar formas de gobierno signadas por restricciones sistemáticas que se identifican en los distintos campos de inserción socio-laboral.

Palabras clave: Intervención Profesional; Trabajo Social; Políticas Públicas; Gubernamentalidad; Neoliberalismo.

Recibido: 31/01/2020 | Aprobado: 18/05/2020

Procedencia del artículo: Artículo derivado del proyecto de investigación Intervenciones sociales del Estado entre los años 2016 - 2019: una lectura a partir de los campos de actuación del Trabajo Social en el Gran La Plata, en las tensiones entre la redistribución y la restricción (IETSYS. FTS. UNLP), acreditado en el marco del Programa de Incentivos del Ministerio de Educación de la Nación, radicado en UNLP (Proyecto T094). 
Danel, Velurtas y Favero-Avico

\title{
Neoliberal Governance: a View from the Interventions of Social Work in Gran La Plata, Argentina
}

\begin{abstract}
In the present work, we share some advances of our research linked to the social interventions of the State between the years 2016-2019, from different fields of action of Social Work in Gran La Plata, Buenos Aires, Argentina. The category of intervention assumes a central role in this study, such that it helps structure the inquiries undertaken about the relation between intervention and social policies.

In recent years, we have developed different research actions aimed at capturing the dynamics acquired by the relationship between the professional intervention of social workers and social policies in different fields. As part of the results, we note that professional intervention has acquired, according to the narratives of social workers, some singularities that give us clues to characterize forms of government marked by systematic restrictions that are identified in the different fields of socio-labour insertion.

Keywords: Professional Intervention; Social work; Public Policies; Governance; Neoliberalism.
\end{abstract}

Sumario: 1. Introducción, 2. Metodología, 3. Hallazgos, 4. Conclusiones, 5. Referencias bibliográficas. 


\section{Introducción}

En el presente trabajo compartimos avances de nuestra investigación vinculada a las intervenciones sociales del Estado entre los años 2016-2019, a partir de distintos campos de actuación del Trabajo Social en el Gran La Plata, Buenos Aires, Argentina. La categoría de intervención adquiere centralidad en la investigación, en tanto creemos que resulta estructuradora de las indagaciones sostenidas sobre la relación entre ésta y los procesos de elaboración y concreción de las políticas sociales en el contexto actual argentino.

En los últimos años, hemos desarrollado distintas acciones investigativas orientadas a capturar la dinámica que adquiere en la actualidad la relación entre la intervención profesional de los trabajadores sociales y las políticas sociales en diferentes campos (Bourdieu y Waquant, 1995). Como parte de los resultados, advertimos que la intervención profesional ha adquirido, según las narrativas de las y los trabajadores sociales, algunas singularidades que nos dan pistas para caracterizar formas de gobierno signadas por restricciones sistemáticas que se identifican en los distintos campos de inserción sociolaboral.

En primer lugar, presentamos el apartado teórico-metodológico propuesto. En segundo lugar, analizamos algunas de las singularidades identificadas en los campos de actuación socio profesional y las tensiones que subyacen en los mismos. Por último compartimos algunas observaciones finales en torno al reconocimiento de las estrategias profesionales y los modos en que las mismas se enlazan con procesos de lucha frente a este periodo de profundización de las desigualdades.

\section{Metodología}

La investigación mencionada es de tipo cualitativa, lo que nos ha permitido bucear en la trama de significados que se producen en los procesos de producción de lo público, en el que interactúan y disputan trabajadores sociales. El trabajo de campo se desarrolla en el aglomerado urbano Gran La Plata, conformado por los distritos de La Plata, Berisso y Ensenada. Los mismos configuran un espacio territorial que conjuga a tres administraciones municipales que se entraman en la geografía de la capital de la Provincia de Buenos Aires con características socio económicas signadas por la desigualdad (véase Figura 1).

En el período de estudio (2016-2019), los distritos de La Plata y Berisso eran administrados por el mismo partido político (Alianza Cambiemos) que la gobernación de Buenos Aires y el gobierno nacional. El distrito de Ensenada es administrado por el partido justicialista. 
Danel, Velurtas y Favero-Avico

Figura 1. Mapa con el área urbana y las cabeceras de partidos del Gran La Plata (Provincia de Buenos Aires, Argentina).

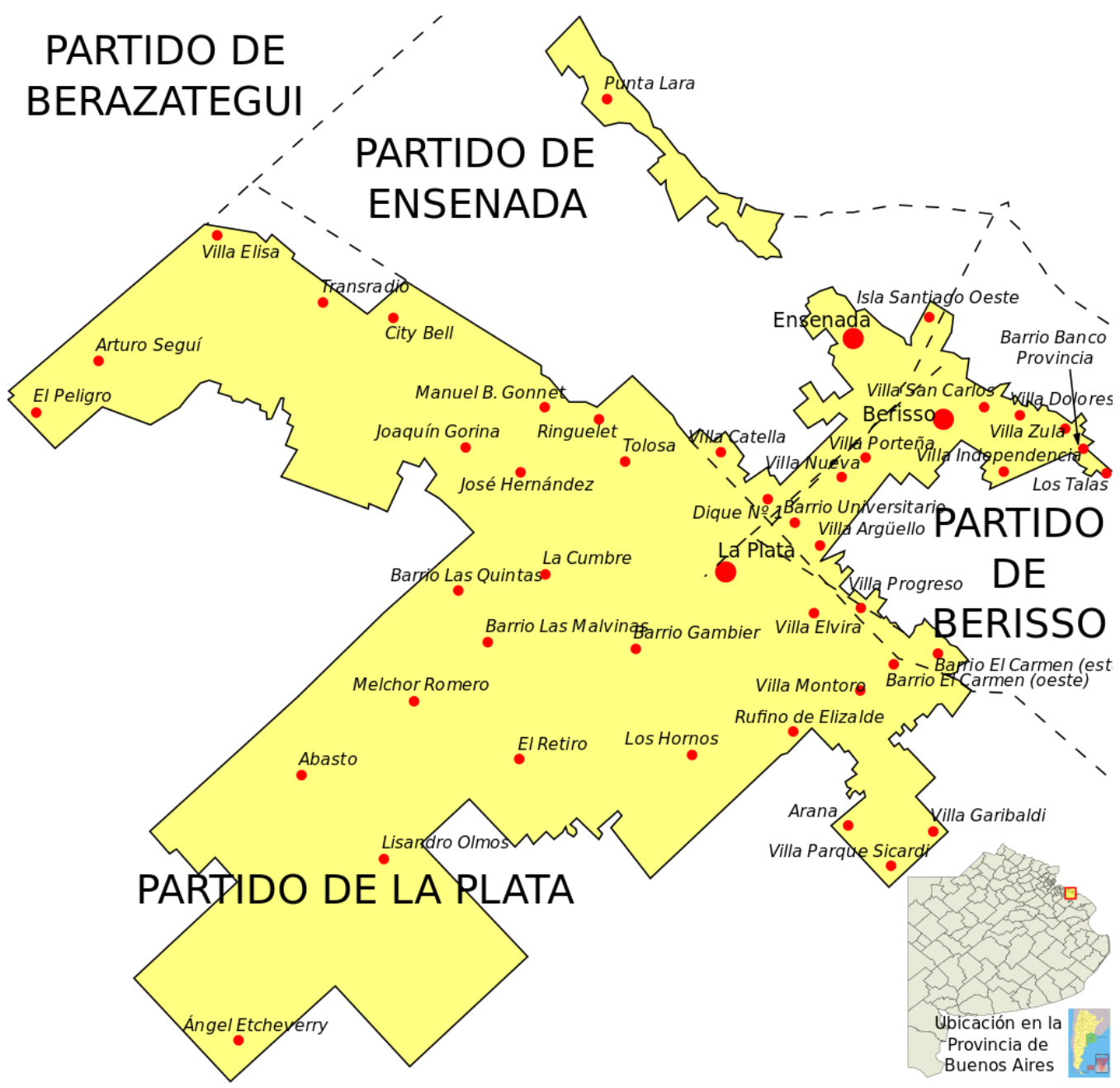

Fuente: Instituto Nacional de Estadística y Censos-INDEC, 2014.

Recuperando antecedentes de otros estudios, señalamos que el conglomerado mencionado presenta sectores con necesidades socio económicas heterogéneas. Esta situación nos permite identificar y reflexionar acerca de la centralidad que le asignamos a la presencia de lo público.

Las necesidades socio-económicas se satisfacen de manera desigual entre el centro de la ciudad de La Plata (véase Figura 2), las periferias y los distritos de Ensenada y Berisso. Estas diferencias emergen en las narrativas de las/os colegas entrevistadas/os, especialmente de aquellas que se desempeñan laboralmente en la órbita municipal. 
Danel, Velurtas y Favero-Avico

Figura 2. Nivel socioeconómico de los hogares del gran La Plata. Distribución según radios censales (CNPHyV 2010)

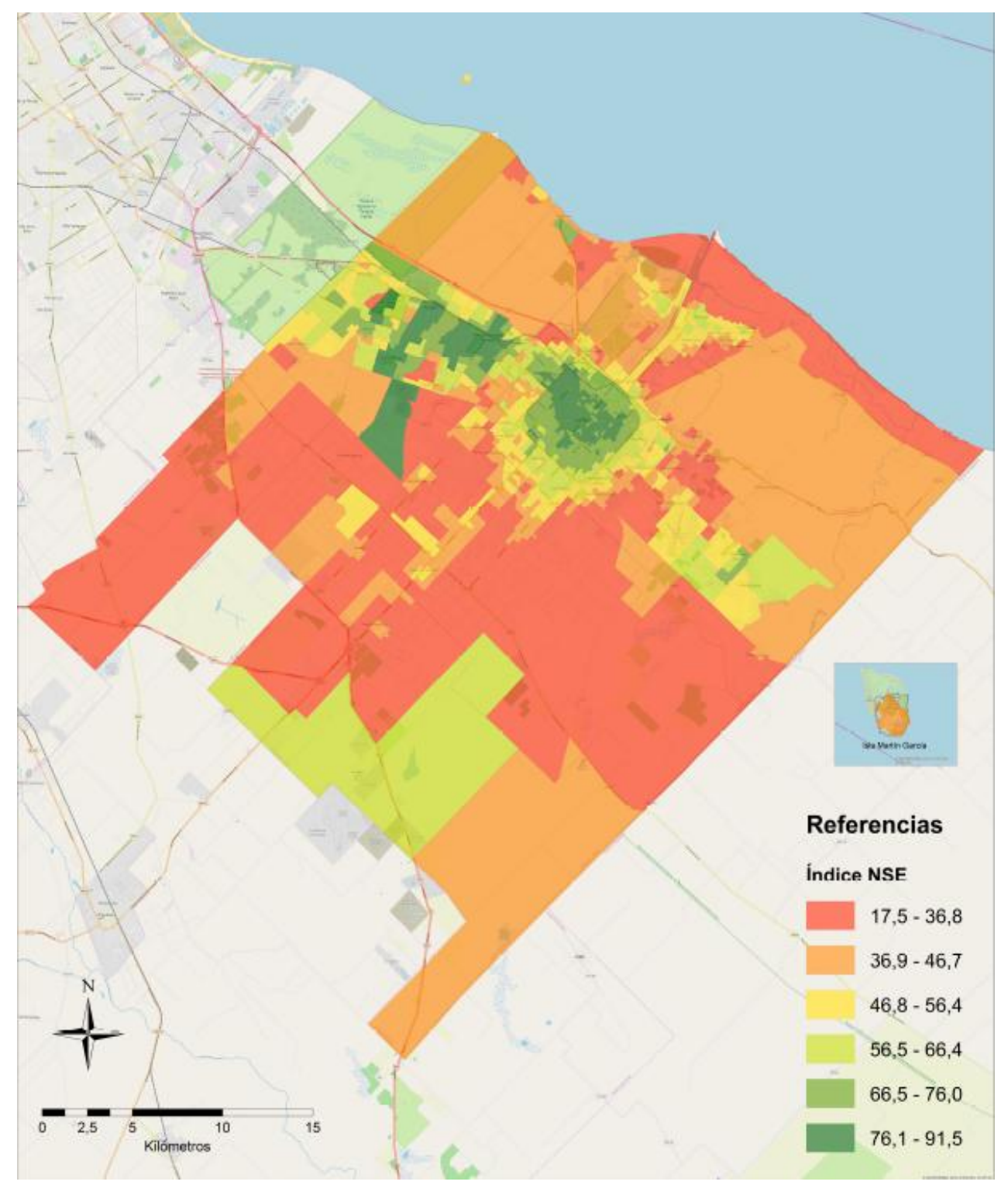

Fuente: Alzugaray, Santa María y Peiró (2019)

En este documento presentamos parte de los resultados de una serie de relevamientos y actividades desarrolladas por el equipo de investigación en el periodo 2016/2019, entre las que destacamos:

- Identificación de características globales de cada campo de intervención, a partir del análisis de fuentes secundarias 
Danel, Velurtas y Favero-Avico

- Entrevistas semi-estructuradas con referentes profesionales del Trabajo Social que se desempeñan actualmente en distintos campos de intervención.

- Grupos focales con referentes profesionales convocados para reflexionar sobre las situaciones que transitan las instituciones en que se insertan como trabajadores y - las condiciones en que despliegan sus intervenciones profesionales, en el contexto reciente.

Estas actividades supusieron el desarrollo de instrumentos de recolección de información, elaborados a partir de una serie de decisiones teóricas signadas por miradas inscriptas en perspectivas posestructuralistas. Siguiendo a Murillo (2011) incluimos la categoría de gubernamentalidad en tanto complejo de tácticas y técnicas desplegadas desde dispositivos que toman a los cuerpos y producen efectos en la construcción y la autoconstitución de sujetos. En los sujetos y sus corporalidades incluimos a los sujetos de las intervenciones profesionales y a los profesionales del Trabajo Social.

Particularmente en este artículo, compartimos una síntesis de las percepciones, claves y argumentos que fueron explicitados por profesionales del Trabajo Social que desarrollan sus prácticas en la zona antes caracterizada. Dichos profesionales fueron convocados a distintas instancias de encuentro personales y grupales para acceder al universo de significados que nos permitan conocer cómo caracterizan y explican el contexto actual, cómo entienden que se configuran sus intervenciones y especialmente el vínculo con las personas con las que se desarrollan intervenciones profesionales.

Uno de los ejes de esta consulta se refirió a los cambios desplegados a partir de la llegada al gobierno nacional, provincial y municipal en la ciudad de La Plata y Berisso de la Alianza Cambiemos y la persistencia del peronismo en el distrito de Ensenada. Destacamos que la masividad que asumió la presencia de la Alianza Cambiemos en la administración nacional, provincial y en dos de los tres distritos de indagación, ha hecho que los contornos de acción se marquen, se empañen con los impulsos que imprimió el "macrismo". Los y las interlocutores/as del trabajo de campo identificaron que desde 2016 se han resignificado las intervenciones profesionales en el marco de los programas sociales y las instituciones en que se insertan. Interlocutores que se encuentran ejerciendo en distintos campos: salud, educación, seguridad social, infancia, juventud y familia, gestión de las políticas sociales; señalan que las transformaciones se desprenden del cambio de orientación política que fue desarrollado bajo la apariencia de un proceso de reorganización administrativa. 
Danel, Velurtas y Favero-Avico

\section{Hallazgos}

Una de las cuestiones destacadas en la investigación, son los modos que para los profesionales del Trabajo Social la intervención adquiere, particularidades que caracterizamos a partir de una nueva gubernamentalidad (Foucault, 1979; O'Malley, 1996). Para los agentes consultados, se trata de un proceso signado por restricciones sistemáticas que emergen en los distintos campos de inserción socio-laboral.

Hemos señalado en artículos recientes (Favero-Avico, Velurtas y Danel, 2019) que desde la llegada al gobierno nacional y provincial de la Alianza Cambiemos, se produjo un viraje sustantivo sostenido en fundamentos ideológicos que se imponen en esta nueva coyuntura. Situación que se traduce rápidamente en severas restricciones presupuestarias y un claro cuestionamiento a la idea de sujeto de derechos que se desplegaba en el periodo que le antecedió. En el contexto señalado observamos procesos orientados a la individuación que toman la forma de una responsabilización. "Cada cual es declarado responsable no solo de su propia suerte...Estos mandatos de individuación encarnan a su vez una exigencia generalizada de "activación" de la voluntad individual" (Castel, Kessler, Merklen y Murard, 2013, p. 47). Estas exigencias, están presentes en la formulación de los objetivos de las políticas sociales y sobrepasan el mero dominio de las mismas, tal como advierten estos mismos autores. Una intervención sobre el otro para transformar a cada sujeto en un individuo de acuerdo con los valores de la autonomía, la responsabilidad y la activación (Merklen, 2013).

Campana (2018) advierte que discutir el derecho a la asistencia social implica necesariamente- discutir su institucionalidad tanto como retomar el debate sobre necesidades y recursos. Para lo cual recupera la noción de precariedad (Butler, 2009), como una condición inducida en la que una serie de personas quedan expuestas al insulto, el estigma, la violencia y la exclusión, con riesgo a ser desprovistas de su condición de sujetos reconocidos ${ }^{1}$. A partir de lo cual, de cómo se define quién es considerado sujeto y quién no, Butler establece una fuerte relación entre precariedad-performatividad-reconocibilidad.

Esta autora caracteriza una condición política inducida de vulnerabilidad maximizada. Se trata de una exposición que sufren las poblaciones que están arbitrariamente sujetas a la violencia del Estado, así como a otras formas de agresión no provocadas por los Estados, pero contra las cuales éstos no ofrecen una protección adecuada. Al hablar de precariedad también alude a personas dedicadas al trabajo sexual y que tienen que defenderse tanto de la violencia callejera como del acoso policial (Butler, 2009).

1 El resaltado es nuestro.

Prospectiva. Revista de Trabajo Social e intervención social • No. 30 • jul.-dic. $2020 \bullet$ pp. 221-238 e-ISSN: 2389-993X • https://doi.org/10.25100/prts.v0i30.8876 
Danel, Velurtas y Favero-Avico

Para la intervención del Trabajo Social, que tradicionalmente ha estado orientada a gestionar procesos de reproducción social, en nuestro país en el último trienio estas transformaciones adquieren particularidades que se asocian con el debate en torno de los límites de los derechos, los umbrales de acceso y las formas de contrataciones de los agentes ya precarizados, ejes que condicionan la relación que estos profesionales establecen con los sujetos de atención.

Desde fines de 2015 o principios del periodo 2016, quienes asumieron la conducción política de los espacios que concentran nuestra atención, inicialmente no plantearon cambios sustantivos, aunque sí importantes recortes presupuestarios en las áreas consultadas. Sin embargo, cuando se analizan los procesos ocurridos en los mismos campos - transcurridos cuatro años-, resulta notable el paulatino y sistemático cercenamiento; se observan distintas manifestaciones que convergen en un embudo signado por las restricciones: la no cobertura de vacantes de personal profesional por jubilaciones, el mantenimiento de los montos establecidos asignados para las asignaciones en dinero, el aumento de requisitos para acceder a programas y recursos, la unificación y centralización de programas, el interés por eludir la intermediación de referentes y profesionales en algunos programas, y el desinterés por fortalecer organizaciones con base territorial, entre los aspectos que destacan los profesionales en todos los campos de distinta manera.

En un período en que el desempleo y empobrecimiento se han incrementado, tal como reflejan las estadísticas privadas y oficiales ${ }^{2}$, estas iniciativas gubernamentales fueron desarrolladas a la par de una serie de cambios normativos que afectaron la organización institucional que, en cada caso, se traducen en el despliegue del abanico de programas, afectando centralmente el acceso a derechos y recursos de la con que los profesionales trabajan.

El diagnóstico compartido en torno a la nueva gubernamentalidad generada a partir de profundos cambios argumentativos en los procesos de producción de lo público, lo pensamos en torno a las estrategias renovadas de ejercicio del poder (Foucault, 1999).Los indicadores nos permiten afirmar que se produce un nuevo entramado, una reconfiguración de dispositivos de intervención que, en clave meritocrática, hace mella en las acciones profesionales.

En esta línea, en los relevamientos y consultas realizadas en el marco de esta investigación, una frase condensó las afirmaciones realizadas por colegas con la cual se tituló una presentación anterior de las autoras: “Uno piensa que va a explotar todo pero

2 Nos referimos tanto al Instituto Nacional de Estadística y Censos (República Argentina) como al Observatorio de la deuda social de la Universidad Católica Argentina. 
Danel, Velurtas y Favero-Avico

hay un vacío total" (Favero-Avico et al., 2019, p. 1). Esta afirmación reúne una serie de situaciones expuestas:

- Notorio empobrecimiento de la población de la ciudad de La Plata y Gran La Plata -usuarios potenciales de estas instituciones- e incremento de percepciones vinculadas al malestar, al sufrimiento social.

- Merma de recursos destinados a la atención de esta población.

- Centralización de oficinas o llamadas "ventanillas" de atención, en espacios alejados de los territorios de relegación urbana.

- Aumento de requisitos para acceder a determinados programas y/o recursos.

- Pérdida de poder adquisitivo de las asignaciones sociales -algunas de las que mantienen el mismo monto desde 2017-.

- Baja de subsidios o beneficios previsionales.

- Falta de cobertura de vacantes de profesionales antes asignados a estas dependencias.

Todas estas situaciones hacen referencia a un contexto en el que la constelación de dificultades se refuerza en un discurso que ha logrado inhibir las demandas sociales, especialmente por el descrédito de su expresión colectiva (Iglesias y Lucca, 2019).

La disminución de demandas en dependencias donde los trabajadores sociales se encuentran insertos, simultáneamente se contrapuso al incremento de conflictividad desplegada en el espacio público de cierto sector del movimiento obrero (especialmente la Central de Trabajadores Argentinos -CTA-). Esta situación paradojal, de reclamo colectivo y presencia disminuida en los circuitos de la institucionalidad estatal, nos da pistas para identificar el modo en que las restricciones en las respuestas se hacen cuerpo en las experiencias vitales de aquellos desventajados. En este marco, se desalienta y estigmatiza a quienes requieren de apoyo y "ayudas", situaciones que se exhiben de manera individual, como el gobierno macrista propone "atenderlas", personas en situación de calle, reaparición de enfermedades asociadas a la pobreza y también el auge de un debate que recorre estos problemas desde la impronta de la seguridad/inseguridad, como ha sido el caso del debate de la baja de la edad de punibilidad en el caso de la justicia.

Retomando la categoría de precariedad desde la perspectiva de la gubernamentalidad, Lorey (2016) identifica que en el marco del proyecto neoliberal, la precariedad se constituye en una novedosa forma de gobierno que impacta en la subjetividad, inscribiéndose en contextos de creciente incertidumbre, inseguridad y descreimiento; modos que proclaman a la precariedad no como un problema a resolverse, sino como un estado que permite repensar y reconducir acciones que impactan, en este caso, en las experiencias vitales tanto de usuarias/os como de profesionales de Trabajo Social. 
Danel, Velurtas y Favero-Avico

A partir de los datos relevados, las/os profesionales entrevistados observan que estas acciones gubernamentales, bajo una apariencia de reforma administrativa, han instaurado obstáculos burocráticos que han provocado el cercenamiento de las posibilidades de acceso a recursos de la población, así como han desmantelado el andamiaje de sostén territorial/comunitario, cerrando dependencias que prestaban servicios en los barrios periféricos de la ciudad de La Plata para concentrarlos en el centro de esta ciudad, lo que ha derivado en el fortalecimiento de otros mecanismos y restricciones de acceso.

Este escenario ha impulsado en la órbita profesional, el fortalecimiento de estrategias de resistencia de las y los colegas, quienes observan que en el espacio territorial han sido escasos los grupos y sectores que han entablado "negociaciones" con las autoridades políticas, a fin de amortiguar el impacto de estas restricciones. En determinados campos de intervención se advierte que una de las formas de resistencia está vinculada a fortalecer los canales de comunicación con otras/os a fin de romper el aislamiento institucional, organizando redes de servicios de Trabajo Social. Asimismo, varios referentes señalaron que en el caso de los trabajadores sociales reaparece un proceso de cuestionamiento a la presencia de estos profesionales: el constante requerimiento de los empleadores de dar cuenta de la tarea cotidiana, justificando el sentido y el quehacer profesional; así como de la necesidad del sostenimiento y constitución de ciertos equipos de interdisciplina. Estos requerimientos son considerados como un esfuerzo extra a la intervención a la que se apuesta.

En este sentido, podemos considerar la vigencia de los aportes de Bourdieu (1999) quien alude a los "pequeños funcionarios" encargados de cumplir las funciones llamadas sociales, esa sensación de ser permanentemente desautorizados en sus esfuerzos por afrontar las situaciones de miseria que provoca la hegemónica lógica de mercado. Agentes que encarnan el mandato estatal vía su presencia en los servicios públicos al tiempo que disponen de medios casi siempre irrisorios, en tiempos de repliegue de la cuestión social.

Autores como Vilas (2011), han planteado el análisis de las intervenciones sociales del Estado “desde arriba y desde abajo”, mientras Gago (2015), repensando la gubernamentalidad en el neoliberalismo, plantea que dichas intervenciones provocan transformaciones "desde arriba, pero en la misma vida cotidiana, desde abajo". Espacio donde el Trabajo Social cuenta con una inserción privilegiada, que nos permite visibilizar expresiones y dilemas que constituyen el desafío de elaboración que nos permita eludir la mera descripción de situaciones individuales, que se imponen en ocasiones a partir de recuperar las crudas experiencias y prácticas profesionales. 
Danel, Velurtas y Favero-Avico

En las interlocuciones con aquellos profesionales que ejercen en el campo de la justicia penal juvenil, advierten una serie de problemas que inciden, producen pérdida de derechos, como por ejemplo: la presencia de las fuerzas de seguridad en centros de régimen cerrados, el empeoramiento progresivo de las condiciones edilicias, dificultades en los traslados de los jóvenes que impiden u obstaculizan su asistencia a las audiencias, el deterioro en la alimentación en cuanto a calidad y cantidad, servicio que el Organismo Provincial de Niñez en la actualidad ha tercerizado. Sumado a ello, se ha incrementado la sobrepoblación y algunas de estas situaciones se han agudizado.

A su turno, en torno a las políticas de cuidado para niñas, niños y adolescentes, otras profesionales advierten sobre el vaciamiento y abandono de espacios y programas de atención del Organismo Provincial de Niñez, como por ejemplo el cierre del Programa de Acompañamiento a la Crianza, Centro Terapéutico Diurno y del Hogar Convivencial "Arrullos", único hogar materno infantil en La Plata. Advierten asimismo cambios en la direccionalidad política de los programas destinados a adolescentes y jóvenes sin cuidados parentales ${ }^{3}$; en tanto que si bien se destaca que con la creación del programa Autonomía Joven y el Programa de Egreso Asistido -a nivel nacional- hubo un avance en términos de acceso a derechos para este grupo. Las profesionales observan la precarización de los programas, en base a ciertos indicadores como el congelamiento del presupuesto y monto de las becas, las condicionalidades y restricciones impuestas para el ingreso a los programas, el desajuste de los tiempos institucionales entre el egreso y el cobro efectivo de las becas, la precarización de los modos de contratación de profesionales y referentes, ausencia de espacios de capacitación y formación continua, así como de supervisión y de discusión de las tareas cotidianas.

De forma paralela, al interior de la órbita judicial se viene implementando otro programa dirigido a jóvenes sin cuidados parentales denominado "Sistema de Sostén" que, según referentes que se desempeñan en el mismo, sufrió profundos cambios en sus objetivos y recorte en sus funciones a partir de 2016, para evitar supuestos solapamientos con el programa del ejecutivo provincial, en tanto éste abarcaría a toda la población adolescente en situación de institucionalización. Las profesionales que intervienen en este campo advierten que en esta reconfiguración, un grupo considerable de jóvenes quedan fuera de ambos programas, por límite de edad en un caso o por la exigencia de condicionalidades para el ingreso y permanencia en otro.

3 Entendemos por adolescentes/jóvenes "sin cuidados parentales" aquellas/os que se encuentran separadas/os de su medio familiar (familia de origen, nuclear y/o extensa o de sus referentes afectivos y/o comunitarios) por haber sido alcanzados por una medida de protección excepcional decidida por el organismo administrativo o judicial ante situaciones de vulneración de derechos y que residen "transitoriamente" en dispositivos de cuidado formal familiar ("de acogimiento") o residencial/institucional (“hogares”), en virtud de dichas medidas (Ley 27364 de 2017, art. 2). 
Danel, Velurtas y Favero-Avico

Considerando las opiniones relevadas, la apelación explícita a la participación de estas instituciones en nombre de la corresponsabilidad en los procesos de inclusión social de niñas, niños, adolescentes y jóvenes, no ha fortalecido al campo de las políticas públicas en esta materia, por cuanto detrás de este principio se encubre el problema de quien asume la responsabilidad por conducción de los procesos. En este sentido, se observan disputas entre el Organismo administrativo y el Poder Judicial sobre quién debe impulsar el proceso de toma de decisiones de las medidas de protección; disputas que se evidencian en condiciones de acceso de los sujetos a los programas e instituciones existentes, dependiendo de qué instancia es la que decide.

Tomamos los aportes de Medan (2019) en sus análisis en torno a los sentidos del riesgo en programas de inclusión social para jóvenes, quien sostiene que:

Las intervenciones, al asumir que parte importante de los riesgos a los que están expuestos los jóvenes provienen de sus familias y sus entornos, no procuran restituir el derecho de los niños a estar en su seno familiar, aun cuando postulen que las instituciones de encierro sean todavía menos convenientes. Así, no construyen una imagen idealizada de la comunidad ni de la familia. Como contra oferta de estos lugares potencialmente riesgosos, los programas, sus sedes, sus trabajadores, sus propuestas vinculares y sus reglas de convivencia buscan ser adoptados por los jóvenes como un lugar propio, como espacio de protección, de distinción y de preparación y adquisición de habilidades para el desarrollo de la autonomía que les permita estar en ese territorio... Si los lineamientos suponen que aprovechando las ayudas de los programas, los jóvenes pueden desarrollar capacidades autónomas para estar preparados a decir no y finalmente no lo logran, la responsabilidad de la falla recaerá en ellos. (p. 166).

Desde esta perspectiva, cuando el planteo institucional parece ponderar, en función del contexto, las posibilidades y los límites de las y los jóvenes, prioriza una tercera locación de los riesgos, en la que relocaliza a ellas y a ellos como esencialmente responsables y/o portadores de esos riesgos.

Los cambios impuestos en este campo, en los modos particulares de gestionar el gobierno de la infancia y juventud, se grafican como un "simulacro", una ficción que implica la creación de programas con una retórica de derechos sociales para este grupo; al mismo tiempo que estos programas presentan una serie de condicionalidades y requisitos que se configuran en barreras de acceso a los mismos, al desarrollarse en un contexto de notable empeoramiento de las condiciones de vida en general, y de las condiciones de vida de los jóvenes durante el tiempo de permanencia en situación de institucionalización en particular (como la presencia de las fuerzas de seguridad en los centros cerrados, la precarización laboral de los equipos de trabajo que dificulta la elaboración de estrategias de egreso, entre otras). Sumado a ello, la falta de acceso a trabajo y vivienda dignos, son 
Danel, Velurtas y Favero-Avico

parte de los problemas más graves a los que este grupo debe enfrentarse y que derivan muchas veces en nuevas vulneraciones de derechos. Estas condiciones, se traducen en dificultades para sostener los proyectos de autonomía una vez concretados los egresos, desdibujando el sentido de los programas.

Por otra parte, en relación al campo educativo, una de las mayores transformaciones que enuncian las/os agentes refiere al enfoque meritocrático que asume la política pública educativa, en referencia a las demandas de intervención a los Equipos de Orientación Escolar, los lineamientos que asumen los programas y la creación de nuevas reglamentaciones ${ }^{4}$. En este sentido, se manifiesta un traspaso de responsabilidades a los niños, niñas, jóvenes y sus familias, apelando a sus esfuerzos individuales como condiciones de acceso al sistema educativo. Asimismo, estos aspectos se expresan en la modificación de la política de educación para adultos, a partir de la creación de las aulas de aceleración, las cuales proponen el cierre de bachilleratos para adultos, problemática que dificulta ampliamente las condiciones de accesibilidad y las intervenciones en materia educativa. Dicha propuesta pone énfasis en la necesidad de enfrentar el problema de la sobre edad, deserción y repitencia desde un enfoque meritocrático que excluye la educación de adultos de las escuelas con una propuesta poco clara en términos curriculares, pedagógicos y laborales. Esta transformación en la educación para adultos impacta en las condiciones de accesibilidad y promoción de la educación ante el cierre de los bachilleratos.

Particularmente en referencia a las intervenciones del Trabajo Social en este campo, los referentes entrevistados identifican que en el último trienio, las habilidades y las competencias individuales se proponen como ejes de intervención principales para pensar lo pedagógico y las trayectorias educativas. En un contexto de aumento de enfoques individualistas que responsabilizan a las familias y a los distintos profesionales, las estrategias de intervención comunitarias se constituyen en una forma de resistencia ante la incertidumbre frente al desafío de garantizar la inclusión educativa en contextos de creciente desigualdad.

La tecnología del poder se despliega en clave de producción de barreras al acceso, con una alta explotación de trabajadores de los organismos. Tal es el caso de las/os profesionales de los organismos de seguridad social, quienes destacan que la estrategia de producción de respuestas institucionales se centra en la eficiencia en los tiempos de atención, desdibujándose la preocupación por el tipo de respuestas brindadas. Se centran en una pronta atención administrativa y se despreocupan de generar acompañamientos a

4 Resolución No. 736 de 2018 del Ministerio de Educación de la República Argentina, Red de Escuelas, Programa Asistiré, Escuelas Promotoras y Programa Nuestra Escuela. 
Danel, Velurtas y Favero-Avico

las trayectorias de las personas. La seguridad social se desdibuja como derecho, y aparece en clave meritocrática. Los cambios en el sistema previsional, operado en el periodo, han generado respuestas diferenciales y restrictivas. La pensión universal del adulto mayor, instala la idea de perceptores legítimos y perceptores que son advenedizos en la seguridad económica.

En el campo de la salud se identificó una tendencia restrictiva, en varios planos:

- El de la estructura organizativa: eliminación del Ministerio de Salud, bajando su estatuto jurídico.

- El de acceso a los dispositivos de apoyo para la sustentabilidad de los tratamientos, lo que originó efectos patológicos agravados,

- En el de las respuestas de las obras sociales, como expresión de la labilidad en la regulación estatal

Surge del análisis del campo de la salud, la producción de desplazamientos de las lógicas del derecho a la de los consumidores, libres y en riesgo.

En torno a las particularidades que adquieren las demandas en el periodo en estudio, destacamos que el conjunto de profesionales advierte preocupación: no hay demanda porque no hay respuesta; ante la ausencia de recursos se comienzan a desarrollar mecanismos de derivación a otras instancias en la búsqueda de recursos y, al mismo tiempo, los programas presentan mayores requisitos. La falta de respuestas desde las organizaciones estatales la analizamos en el marco de una serie de estrategias de subjetividad contemporánea, es decir, como parte de las tecnologías de poder desplegadas para la producción de subjetividad concordantes con el capitalismo contemporáneo.

Esto puede vincularse a la esfera de las restricciones presupuestarias como otro punto de tensión a lo que los profesionales hacen referencia. Estos reconocen que no es posible garantizar la llegada de recursos a las familias, por lo que también desde los equipos subyace una resistencia a generar expectativas, cuando no hay una respuesta institucional sino una situación de vaciamiento, barreras de acceso (Chaves, 2005) a lo asistencial.

\section{Conclusiones}

En el presente trabajo cristalizamos un análisis que persigue sobrepasar relatos de situaciones que signan el universo histórico del Trabajo Social: anécdotas que emergen a diario en relatos recortados, en la queja, el padecimiento compartido, la catarsis aislada que se integran en cada contexto como una constelación. La búsqueda estuvo asociada a pensar desde las prácticas profesionales, cómo se hegemonizaron los modos estatales de intervención en el período 2016-2019, en el Gran La Plata. 
Danel, Velurtas y Favero-Avico

Las colegas con las que se interactuó compartieron sus pareceres, sus miradas y sus análisis sobre las formas que asumió la nueva gubernamentalidad. La misma -de manera creciente- generó restricciones que impregnaron las instituciones, los dispositivos. A su turno, a excepción del campo de la justicia, las condiciones de contratación ya precarias han agudizado inseguridad a la situación laboral de los profesionales.

En todos los casos se registra malestar con el nuevo entramado estatal, con el lugar que se le asigna a la profesión y destacan sobre asignación de tareas. Ello ocurre simultáneamente con un proceso de empobrecimiento generalizado de la población usuaria de estos servicios y programas. Tal situación, generó distintas estrategias de los profesionales que tensan la noción de "estrategias de atrincheramiento" (Andrenacci citado en Campagna, 2017). Creemos que si bien se tendió a un trabajo puertas adentro de las organizaciones, en un contexto de persecución política y despidos, el horizonte era abrir los espacios. Si fueran trincheras marcarían la imagen de "zanja defensiva que permite disparar a cubierto del enemigo" y las colegas defendían los espacios para que sean habitados. Frente al vacío en las respuestas, las colegas generaron estrategias de fortalecimiento interno y de los lazos con los sujetos usuarios de los servicios y programas.

Las instituciones en el neoliberalismo, refuerzan el descrédito de sujetos y dispositivos estatales que renovaron las formas de gobernar y ejercer poder sobre una determinada población. En este sentido, la gubernamentalidad neoliberal, representa un aspecto nodal en nuestro análisis para reflexionar acerca de estos tiempos estatales, constituyéndose en un campo en tensión en el que se insertan los trabajadores sociales. Un escenario que entendemos se caracteriza por la profundización de las desigualdades y en donde reafirmamos como fundamento de la intervención profesional del Trabajo Social el concepto de cuestión social (Rozas-Pagaza, 2018).

Siguiendo esta línea analítica, entendemos que en el período estudiado asistimos a procesos en los que se redefinieron las condiciones en que se despliegan las intervenciones profesionales. Esto supuso disputas, confrontaciones que se materializaron en la cotidianidad de las organizaciones estatales. Identificamos sentidos, significados y reorientaciones que provocaron multiplicidad de tensiones que moldearon los espacios de acción posible.

La velocidad y el impacto de estas transformaciones y la capacidad de los agentes para afectar los procesos de intervención en los distintos campos se constituyen como un nudo central de problemas. Las estrategias desplegadas se diferencian entre sí, algunos pensaron en procesos de singularización, otros en potenciarse vía la conformación de redes. Las formas de resistencia son diversas en cuanto a cómo se han ido reconfigurando las distintas instancias estatales o dispositivos de atención en que se insertan. 
Danel, Velurtas y Favero-Avico

Desde la perspectiva presentada asumimos que las políticas constituyen un campo de disputa de significados, por lo que existen modos diferenciales de habitarla. La referencia a la resistencia desplegada por los/as trabajadores/as sociales nos pone en diálogo con Deleuze (1996) quien afirma que las actividades intelectuales (entre las que inscribimos el Trabajo Social) suponen la producción de conceptos (lectura situacional y operación sobre la misma) lo que tiene fuerza crítica de liberación. De algún modo, Deleuze (1996) señala que crear conceptos es resistir.

En tanto que la gubernamentalidad neoliberal persigue el ocultamiento de las desigualdades sociales a partir de intervenciones clasificatorias de las necesidades sociales y de la fragmentación de lo social, profundizando el estado de precariedad de los sujetos con los que trabajamos, entendemos que los trabajadores sociales, podemos contribuir a reproducir o a transformar desde nuestras prácticas profesionales, como opción éticopolítica, esta condición.

Resulta necesario mostrar la lógica de la actual acumulación del capital globalizado y cómo dichas desigualdades se van profundizando comprometiendo las bases de su propia existencia. Se trata de saber en qué lugares de esos mecanismos de gobierno cabe encontrar fisuras, capacidades y resistencia.

\section{Referencias bibliográficas}

Alzugaray, L., Santa María, J., y Peiró, M. (2019).Cartografía del nivel socioeconómico de los hogares del Gran La Plata: Aplicación de un indice a partir de datos del Censo 2010. Ensenada: Universidad Nacional de La Plata. Recuperado de http:/ / www.memoria.fahce.unlp.edu.ar/library?a=d\&c=libros\&d=Jpm1070.

Bourdieu, P. (1999). Razones prácticas, sobre la teoría de la acción. Barcelona: Anagrama.

Bourdieu, P., y Waquant, L. (1995). Respuestas. Por una antropología reflexiva. México: Grijalbo.

Butler, J. (2009). Lenguaje, poder e identidad. Madrid: Editorial Síntesis.

Campagna, M. (2017). Asistencia Social y restauración neoliberal. Cátedra Paralela, (14), 13-24.

Recuperado

de

http:/ / catedraparalela.com.ar/revistasoficial/revista14/sumario.pdf.

Campana, M. (2018). La Asistencia Social como derecho: integración y precariedad. Escenarios, 18(27), 1-8. Recuperado de https://revistas.unlp.edu.ar/escenarios/article/view/8084.

Castel, R., Kessler, G., Merklen, D., y Murard, N. (2013). Individuación, Precariedad, Inseguridad: ¿desinstitucionalización del presente? Buenos Aires: Paidós.

Chaves, M. (2005). Juventud negada y negativizada. Representaciones y formaciones discursivas vigentes en la Argentina Contemporánea. Última Década, 13(23), 9-32. 
Danel, Velurtas y Favero-Avico

Congreso de la Nación Argentina. (26 de junio de 2017). Artículo 2. [Título I]. Programa de acompañamiento para el egreso de jóvenes sin cuidados parentales. [Ley 27364]. BO: 26/06/2017.

Deleuze, G. (1996). Conversaciones (1972-1990). Valencia: Pre-Textos.

Favero-Avico, A., Velurtas, M., y Danel, P. (septiembre, 2019). Uno piensa que va a explotar todo pero... hay un vacío total. En XI Jornadas de Investigación, Docencia, Extensión y Ejercicio Profesional: "Disputas por el Estado, la democracia y las políticas públicas. Concentración de la riqueza y poder popular. Universidad Nacional de La Plata, Facultad de Trabajo Social, La Plata, Argentina. Recuperado de http:/ / sedici.unlp.edu.ar/handle/10915/94134.

Foucault, M. (1979). Governmentality. Ideology \& Consciousness, 6, 5-21.

Foucault, M. (1999). La arqueología del saber. México: Siglo XXI editores.

Gago, V. (2015). La razón neoliberal. Economías Barrocas y pragmática popular. Buenos Aires: Traficante de Sueños.

Iglesias, E., y Lucca, J.B. (Comps.). (2019). La Argentina de Cambiemos. Argentina: UNR Editora. Recuperado de https://rephip.unr.edu.ar/xmlui/bitstream/handle/2133/15433/ArgentinadeCam biemos.pdf? sequence $=3 \&$ isAllowed $=\mathrm{y}$.

Instituto Nacional de Estadística y Censos-INDEC. (2014). Censo Nacional de Población, Hogares y Viviendas 2010. Censo del Bicentenario. Serie C. Población con dificultad o limitación permanente. Buenos Aires: INDEC.

Lorey, I. (2016). Estado de inseguridad. Gobernar la precariedad. España: Traficante de Sueños.

Medan, M. (2019). El territorio, la comunidad y la autonomía: ¿discursos mitológicos en los programas sociales destinados a jóvenes "en riesgo". Ciudadanías. Revista de Políticas Sociales Urbanas, (4), 139-170. Recuperado de http://ciudadanias.untref.edu.ar//n4_dossier_art6.php.

Merklen, D. (2013). Las dinámicas contemporáneas de la individuación. En R. Castel, G. Kessler, D. Merklen y N. Murard, Individuación, Precariedad, Inseguridad: ¿desinstitucionalización del presente? (pp.45-86). Buenos Aires: Paidós.

Ministerio de educación, cultura, ciencia y tecnología de la Nación Argentina. (10 de octubre de 2018). [Resolución 736 de 2018]. BO: 16/10/2018.

Murillo, S. (2011). Estado, sociedad civil y gubernamentalidad neoliberal. Entramados y perspectivas, 1(1), 91-108.

O’Malley, P. (1996). Risk and responsibility. En A. Barry, T. Osborne y N. Rose (Eds.), Foucault and Political Reason: Liberalism, Neo-Liberalism, and Rationalities of Government (pp. 189-209). London: University of Chicago Press. 
Danel, Velurtas y Favero-Avico

Rozas-Pagaza, M. (octubre, 2018). La cuestión social y su relación con el concepto de precariedad. En VIII Conferencia Latinoamericana y Caribeña de Ciencias Sociales. Eje 9 Desigualdades y Pobreza, Panel 25, Buenos Aires, Argentina.

Vilas, C. (2011). Política y Políticas Públicas en América Latina. En C. Fioramonti y P. Anaya (Comps.), El Estado y las políticas públicas en América Latina (pp. 37-74). La Plata: AECID/COPPPAL/Honorable Cámara de Senadores de la Provincia de Buenos Aires. Recuperado de http://cmvilas.com.ar/index.php/articulos/12politicas-publicas/27-politica-y-politicas-publicas-en-america-latina. 


\section{OTROS ARTÍCULOS DE PROSPECTIVA No. 30 DE 2020}

\section{EDITORIAL}

Coherencia, integridad y vida cotidiana

Luz Mary Sánchez-Rengifo

\section{ARTÍCULOS}

Trabajo Social y medios de comunicación: perspectivas y posibilidades de encuentro Social

Mercedes Muriel-Saiz

Maribel Martín-Estalayo

Seguridad, afectos y familias. Obstáculos en el proceso de reintegración de personas desmovilizadas de grupos

armados en Santander, Colombia

Jakeline Vargas-Parra

Ángela María Díaz-Pérez

Priscyll Anctil-Avoine

Reflexividad sobre la intervención profesional en duelo con población afectada por el conflicto armado en Colombia

María Cénide Escobar-Serrano

Maritza Charry-Higuera

Natalia Ramírez-Moncada

Ser mujer indígena, náhuatl, casada, migrante, sin trabajo remunerado: una realidad en los albergues jornaleros agrícolas en Colima, México

Nancy Elizabeth Molina-Rodríguez

Tipos y manifestaciones de la violencia de género: una visibilización a partir de relatos de mujeres víctimas en Soacha, Colombia

Diana Carolina Tibaná-Ríos

Diana Alejandra Arciniegas-Ramírez

Ingrid Julieth Delgado-Hernández

Análisis de necesidades en familias monoparentales con jefatura femenina usuarias de servicios sociales de atención primaria en España

Celia María Fernández-Martínez

Manuela Avilés-Hernández

Experiencias de intervención de trabajadoras sociales con trabajadores sexuales masculinos en Bogotá, Colombia Johan Arturo Barrera-Castellanos
Condiciones laborales de trabajadores sociales en hospitales públicos en la provincia de Mendoza,

Argentina

Cecilia Amalia Molina

Yanina Noemi Roslan-Angeloni

Analía Graciela Correa

Viviana Elena Varela

Gubernamentalidad neoliberal: miradas desde las intervenciones del Trabajo Social en el Gran La Plata, Argentina

Paula Mara Danel

Marcela Claudia Velurtas

Agustina María Favero-Avico

Educación superior en Trabajo Social en Chile y formación para la intervención en situación de calle. Desafios desde la evaluación que interventores hacen de sus procesos de práctica pre-profesional

Carlos Alejandro Andrade-Guzmán

Ignacio Andrés Eissmann-Araya

Educación en Derechos Humanos para el Trabajo Social en Chile: una mirada desde los estándares

internacionales

Lury Soledad Reyes-Pérez

Vivianne Soledad Hasse-Riquelme

Luis Marcelo Silva-Burgos

Arriesgar y preservar la vida: derechos humanos, conflicto sociopolítico armado y Trabajo Social en Colombia

Cristian Sebastián Castaño-Orozco

Ricardo Patiño-Martínez

\section{IN MEMORIAM}

Cristina Bautista Taquinás. Mujer indígena Nasa, Trabajadora Social, lideresa del norte del Cauca, Colombia

Alba Nubia Rodríguez-Pizarro

Lady Johanna Betancourt-Maldonado
ARTISTA INVITADO
MALA JUNTA KLAN
Alejandra Gutiérrez-Cárdenas

\section{PROSPECTIVA}

